\title{
Influence of $\beta$-galacto-oligosaccharide on growth performance and components of intestinal barrier in broilers during heat stress
}

\author{
S. Ashraf ${ }^{1}$, H. Zaneb ${ }^{1 \#}$, S. Masood ${ }^{1}$, S. Yousaf ${ }^{2}$, M. M. Usman', H. F. Rehman ${ }^{1}$, A.Sikandar ${ }^{3}$ \& \\ H. Rehman ${ }^{2}$ \\ ${ }^{1}$ Department of Anatomy and Histology, University of Veterinary and Animal Sciences, 54000, Lahore, Pakistan. \\ ${ }^{2}$ Department of Physiology, University of Veterinary and Animal Sciences, 54000, Lahore, Pakistan \\ ${ }^{3}$ Department of Anatomy and Histology, Sub-campus, Jhang, University of Veterinary and Animal Sciences, Lahore
}

(UVAS), 35200, Pakistan

(Received 19 April 2017; Accepted 21 June 2017; First published online 7 August 2017)

Copyright resides with the authors in terms of the Creative Commons Attribution 4.0 South African License.
See: http://creativecommons.org/licenses/by/4.0/za
Condition of use: The user may copy, distribute, transmit and adapt the work, but must recognize the authors and the South African
Journal of Animal Science.

\begin{abstract}
This study aimed to investigate the influence of $\beta$-galacto-oligosaccharides ( $\beta$-GOS) on growth performance, organ development and intestinal microarchitecture of broilers during heat stress. Day-old chicks $(n=125)$ were divided into five groups. The control or thermoneutral zone group (TNZ) was raised under standard management until the 35th day. Four groups were exposed to cyclic heat stress $\left(35^{\circ} \mathrm{C} 8 \mathrm{~h} / \mathrm{d}\right)$ from the 22nd to the 35th day. The TNZ and heat stress control (HSCT) groups were fed a corn-based diet and HS + $0.1 \% \beta$-GOS; HS + $0.2 \% \beta$-GOS; and HS + $0.5 \% \beta$-GOS were fed a corn-based diet supplemented with $\beta$-GOS $(0.1 \%, 0.2 \%$, and $0.5 \%)$, respectively. Exposure to heat stress reduced feed consumption, feed efficiency and the relative weight of the liver, bursa of Fabricius, and small intestine, compared with the TNZ group. Morphometric evaluation of the small intestine revealed reduced villus surface area, villus height to crypt depth ratio (VH : CD) and intraepithelial lymphocytes (IELs) in all segments, and reduced acidic goblet cells (AGCs) in the ileum of the HSCT group compared with the TNZ group. Compared with the HSCT group, dietary $\beta$-GOS $(0.2 \%$ and $0.5 \%)$ improved the feed efficiency and relative weight of the small intestine. Furthermore, dietary $\beta$-GOS $(0.1 \%)$ increased villus surface area in the duodenum and IEL count in the small intestine compared with the HSCT group. Dietary $\beta$-GOS $0.5 \%$ increased villi surface area (VSA) in the jejunum and ileum, whereas the IEL count in the small intestine and acidic goblet cells (AGCs) in the jejunum and ileum were reduced compared with the HSCT group. In conclusion, dietary supplementation of $\beta$-GOS $(0.2 \%$ and $0.5 \%)$ improved the growth performance and intestinal microarchitecture of broilers during heat exposure, along with partial immune stimulation.
\end{abstract}

Keywords: Feed efficiency, goblet cell, intraepithelial lymphocytes, mucosal architecture, prebiotics, poultry, villus morphometry

\#Corresponding author: hafsa.zaneb@uvas.edu.pk

\section{Introduction}

Intensive selection of broilers for growth rate and feed efficiency not only imposes high demands on their metabolic processes (Decuypere et al., 2000), but increases their sensitivity to thermal stress (Burkholder et al., 2008). For optimal growth and production, broilers must be raised in their thermoneural zone, but in Pakistan environmental temperatures during the summer months can reach $52{ }^{\circ} \mathrm{C}$ (Kazmi et al., 2015). Although controlled poultry sheds are equipped with temperature-mitigating devices, temperature maintenance by them is costly (Yoo et al., 2016). Moreover, severe energy crises in summer make it more difficult to maintain the temperature of the shed. Broilers exposed to heat stress exhibit poor weight gain, endocrine disturbances, immunosuppression, gastrointestinal damage, microbial dysbiosis, and high mortality. Apparent adaptations during heat stress include reduced feed intake and mobility, with a concomitant increase in drinking water and panting, as reviewed by Lara \& Rostagno (2013). These behavioural modifications are coupled with redirection of blood flow to the periphery. The resulting hypoxia in the gut not only damages the villus enterocytes (Song et al., 2014) and goblet cells (Olsen et al., 2005), but also induces the recruitment of intra epithelial lymphocytes (IELs) (Quinteiro-Filho et al., 2010). These perturbations of the mucosal barrier are not limited to the microarchitecture, but involve its microbial 
compartment. Microbial dysbiosis during heat stress predisposes the birds to gastrointestinal infections such as salmonellosis and coccidiosis, which are responsible for morbidity, mortality and loss of productivity (Burkholder et al., 2008).

Production losses during thermal stress were reduced in poultry by the use of antibiotic growth promoters (Huff et al., 2015). But with the emergence of antibiotic-resistant superbugs and antibiotic residues in meat, their use in animal feed was banned by the European Commission in 2006 (EC Regulation No. 1831/2003). Antibiotic withdrawal greatly increased the incidence of clinical infections and the use of therapeutic antibiotics in broilers. Thereafter, it was realized that a simple ban on these growth promoting antibiotics would not offer a solution to the problem until alternative strategies were planned to improve the health and efficiency of broilers, particularly during stressful situations (Alloui et al., 2013). As an alternative to these antibiotic growth promoters, a wide range of products gained the attention of researchers, the most widely studied and acclaimed agents being probiotics and prebiotics. Prebiotics are non-digestible oligosaccharides that enrich the innate microbial ecosystem of the host. The property of prebiotics to promote the naturally adapted beneficial flora of the host makes them a superior choice to probiotics (Callaway et al., 2012; Alloui et al., 2013).

Galacto-oligosaccharide is a low-priced prebiotic agent that is used extensively. It is one of three prebiotics that meet the criteria of the European Union (Kolida \& Gibson, 2011). The lacto-bifidogenic property of $\beta$-galacto-oligosaccharides ( $\beta$-GOS) is widely acclaimed in humans and animals, and provides a basis for the improvement observed in gastrointestinal health, structure and immune function (BrunoBarcena \& Azcarate-Peril, 2015). Nevertheless, $\beta$-GOS, which originated from Bifidobacterium galactosidase, failed to improve the performance of broilers in thermoneutral situations. But it has been suggested that the effects of prebiotics on the performance of broilers are variable (Biggs et al., 2007). Little information exists regarding the role and efficacy of $\beta$-GOS in broilers during heat stress (Varasteh et al., 2015), particularly with reference to growth performance and intestinal barrier components. Therefore the present study was planned to elucidate the role of $\beta$-GOS from Lactobacillus reuteri L103 galactosidase with predominant $\beta-(1 \rightarrow 6)$ and $\beta-(1 \rightarrow 3)$ linkages on growth performance, organ development, mucosal architecture, histochemistry, and differentiation of goblet cells and count of IELs in the small intestines of broilers during cyclic heat stress.

\section{Materials and Methods}

This study was carried out in a research shed at the University of Veterinary and Animal Sciences (UVAS), Lahore. The plan was approved by the Ethical Review Committee for the Use of Laboratory Animals, and Directorate of Advanced Studies, UVAS (Notification No. DAS/517 dated 25-3-14). This experiment lasted 35 days, and was conducted on 125 day-old Hubbard broiler chicks obtained from a commercial hatchery. On arrival, the birds were weighed and randomly assigned to five groups (TNZ, HSCT, $\mathrm{HS}+0.1 \% \beta$-GOS; HS + 0.2\% $\beta$-GOS; and HS + 0.5\% $\beta$-GOS) with five replicates in each group. The floor was covered with wood shaving litter. Feed (starter and grower mash) and water were offered ad libitum. All diets were formulated without antimicrobials and coccidiostats according to NRC (1994) recommendations (Table 1). On day 1, ambient temperature and relative humidity were maintained at $35{ }^{\circ} \mathrm{C} \pm 65 \%$ respectively. However, a weekly decrease $\left(2.8^{\circ} \mathrm{C}\right)$ in temperature was done to attain a thermoneutral zone, that is, $26{ }^{\circ} \mathrm{C} \pm 65 \%$ relative humidity at day 21 . On day 22 , one group was kept at a thermoneutral zone, which served as control (TNZ). The remaining four groups were exposed to cyclic temperature treatment of $35{ }^{\circ} \mathrm{C}$ for 8 hours during the daylight period and $26^{\circ} \mathrm{C}$ for the remaining period. These four groups were termed heat stress groups. The TNZ and the heat stress control group (HSCT) were fed a standard cornbased diet, whereas the three heat stress groups (HS + 0.1\% $\beta$-GOS; HS + 0.2\% $\beta$-GOS; and HS $+0.5 \% \beta-$ GOS) were fed the standard diet supplemented with dietary levels of $\beta$-GOS. Birds were vaccinated intraocularly by live attenuated Newcastle disease virus (Ceva-Phylaxia, Budapest, Hungary) on day 1, with a booster on day 20 in drinking water. Similarly, vaccination against infectious bronchitis disease (Lohman Animal Health $\mathrm{GmbH}$, Cuxhaven, Germany) was done by the intraocular route on day 8 and repeated on day 20 in the drinking water.

The $\beta$-GOS mixture was prepared by the Department of Food Science and Human Nutrition, UVAS, Lahore. It was produced by $\beta$-galactosidase from Lactobacillus reuteri $L 103$, employing lactose as a substrate. The resultant mixture of $\beta$-GOS, which possessed a degree of polymerization (dp) of $2-3$, contained $\sim 80 \%$ of $\beta-(1 \rightarrow 6)$ and $\sim 9 \% \quad \beta-(1 \rightarrow 3)$ linked transgalactosylation products of the total GOS, whereas $\beta-(1 \rightarrow 4)$-linked products could not be detected. The main components of GOS mixtures were $\beta$-D-

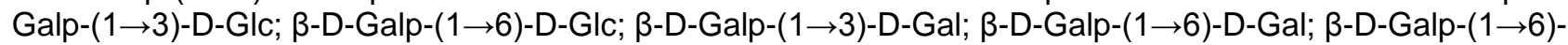

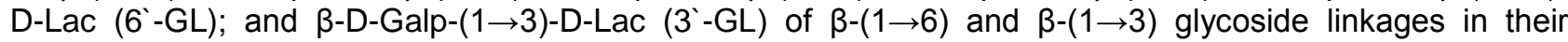
transgalactosylation products. When the galactosidase from Lactobacillus reuteri L103 is utilized for GOS production, $\beta-(1 \rightarrow 6)$-linked products constitute $60 \%$ of the total linkages formed during the reaction. 
Table 1 Feed ingredients and proximate composition of the starter (1-21 days of age) and grower diets (22-35 days of age) for broilers.

\begin{tabular}{lrr}
\hline Ingredient (g/kg) & Starter phase & Grower phase \\
\hline Corn & 401.5 & 575.7 \\
Rice broken & 150 & --- \\
Soy meal & 115.4 & 96 \\
Sunflower meal & 120 & 130 \\
Canola meal & 90 & 50 \\
Rapeseed meal & 50 & 76 \\
Rice polish & --- & 40 \\
Guar meal & 10 & --- \\
Wheat bran & 13.4 & --- \\
Molasses & 20 & -- \\
Sodium bicarbonate & 0.3 & 0.65 \\
Sodium chloride & 2.1 & 2.1 \\
Di-calcium phosphate & 13.3 & 14.9 \\
L-Lysine & 3 & 3.5 \\
DL-Methionine & 1 & 1.2 \\
Vit-mineral premix* & 10 & 10 \\
Nutrient composition & & \\
Calculated ME (kcal.kg) & & 2850 \\
CP & 2750 & 185 \\
DM & 196 & 880 \\
Crude fibre & 870 & 63.5 \\
Crude fat & 60.5 & 23.5 \\
Total ash & 21.6 & 54 \\
\end{tabular}

* Vitamin mineral premix (per kg ): ascorbic acid 26000 IU; retinol, 200000 IU; cholecalciferol 80,000 IU; tocopherol $1072 \mathrm{IU}$; thiamine $11666 \mathrm{IU}$; pyridoxine $33333 \mathrm{IU}$; menadione 11,333 IU; riboflavin 54,000 IU; niacin 5,36,000 IU; folic acid $13600 \mathrm{IU}$; methylcobalamin $223 \mathrm{IU}$; biotin $1340 \mathrm{IU}$; Ca 195 g; K 70 g; Na 18 g; Mg 6 g; Fe 2000 mg; Zn 1200 mg; Mn 1200 mg; Cu 400 mg; I 40 mg; Co 20 mg; Se 8 mg

ME: metabolizable energy; CP: crude protein; DM: dry matter

For measuring the growth parameters of broilers, feed consumption and bodyweight were recorded weekly. Feed consumption was calculated daily by deducting the feed left in the feeders from the feed offered. To calculate feed efficiency, bodyweight was divided by the feed consumption of the birds.

On day 35 , ten birds per treatment (two birds/replicate) were randomly selected and slaughtered by severing the jugular vein. After weighing the birds, the viscera, including liver, pancreas, spleen, heart, bursa, gizzard and small intestine (duodenum, jejunum, and ileum), were collected and weighed. Absolute weight of these viscera was used to calculate the relative organ weight.

Approximately two-centimetre-long samples were taken from the mid portions of the duodenum, jejunum and ileum. The samples were flushed with normal saline and placed in $10 \%$ neutral buffered formalin. Intestinal segments were processed by routine paraffin embedding (Bancroft et al., 2013), sectioned (Amos Scientific AEM-450, Austria) to obtain a segment of $5 \mu \mathrm{m}$, and stained with two techniques for observing the mucosal morphology, IELs and goblet cells.

Small intestinal sections that were subjected to routine haematoxylin and eosin (H\&E) staining (Bancroft et al., 2013) were observed for villus height, villus width and crypt depth. Villus height ratio crypt depth (VH:CD) was also calculated. In a section, villi were selected based on intact lamina propria, and all observations were taken on five well-oriented villus crypt units. Villus height was taken from the top of the villus to the interface with the crypt origin. Crypt depth was measured from origin to base, and villus width 
was taken from the middle of the villus. All measurements of morphometric parameters were taken in $\mu$ m. Slides were observed with a light microscope (Labomed, Lx 400, Fremont, Calif, 9453, USA) equipped with a digital imaging system and a commercial morphometry program. Villi surface area was calculated using the (2 $\pi$ ) (villus width/2) (villus height) formula (Solis de los Santos et al., 2007). The Intra epithelial lymphocytes count was performed on H\&E stained slides. In total, five villi were observed for counting IELs. Avian IELs are described as round cells within the epithelium of villi. They possess spherical, heterochromatic nuclei surrounded by scant cytoplasm (Thrall et al., 2004). These IELs are readily distinguishable from the oval nuclei of enterocytes in the intestinal mucosa.

Tissue sections prepared with the paraffin-embedding technique were subjected to the combined alcian blue-periodic acid Schiff (AB-PAS) staining method for observing acidic mucin containing goblet cells, as described by Bancroft et al. (2013). Acidic mucin goblet cells are described on the basis of their glycoprotein content. Five villi per section were observed with a light microscope to count various types of goblet cells.

The data were presented as mean \pm standard error (SE). Normal distribution of the data was checked with the Kolmogrov Smirnov test. Statistical differences among treatment groups were analysed with SPSS for Windows version 20.0 (SPSS Inc., Chicago, II, USA). Data analysis was done with one-way ANOVA. Statistical differences among means were identified with Duncan's multiple range tests and considered significant at $(P<0.05)$.

\section{Results}

The results in growth performance in response to heat stress and supplementation of $\beta$-GOS are presented in Table 2. Heat exposure lowered $(P<0.05)$ bodyweight and feed efficiency during the fourth week and feed consumption, bodyweight, and feed efficiency during the last week in broilers of the HSCT group compared with the TNZ group. During the fourth week, supplementation of $\beta$-GOS improved $(P<0.05)$ bodyweight in $\mathrm{HS}+0.5 \% \beta$-GOS, and feed efficiency in $\mathrm{HS}+0.2 \% \beta$-GOS and $\mathrm{HS}+0.5 \% \beta$-GOS groups compared with the HSCT group. Similarly, during the last week, feed consumption, bodyweight, and feed efficiency were better $(P<0.05)$ in $\mathrm{HS}+0.1 \% \beta$-GOS, $\mathrm{HS}+0.2 \% \beta$-GOS and $\mathrm{HS}+0.5 \% \beta$-GOS groups, compared with the HSCT group (Table 2).

Table 2 Effects of heat stress and $\beta$-galacto-oligosaccharides on growth parameters in broilers

\begin{tabular}{|c|c|c|c|c|c|c|c|c|}
\hline \multirow{2}{*}{ Parameter } & \multirow{2}{*}{ Week } & \multicolumn{5}{|c|}{ Groups* } & \multirow{2}{*}{$\begin{array}{l}\text { Pooled } \\
\text { SEM }\end{array}$} & \multirow{2}{*}{$\begin{array}{c}\text { P. } \\
\text { value }\end{array}$} \\
\hline & & TNZ & HSCT & HS+0.1\% $\%$-GOS & HS+0.2\% $\%$-GOS & HS+0.5\% $\%-G O S$ & & \\
\hline \multirow{3}{*}{$\mathrm{FC}(\mathrm{g})$} & 3rd & 857 & 863 & 858 & 865 & 876 & 7.5 & 0.40 \\
\hline & 4 th & $1624^{a}$ & $1300^{\mathrm{b}}$ & $1646^{a}$ & $1657^{\mathrm{a}}$ & $1563^{a}$ & 23 & 0.02 \\
\hline & 5 th & $2581^{\mathrm{a}}$ & $2238^{c}$ & $2639^{a}$ & $2405^{\mathrm{b}}$ & $2604^{\mathrm{a}}$ & 37 & 0.04 \\
\hline \multirow{3}{*}{$\begin{array}{l}\text { BW } \\
\text { (g) }\end{array}$} & 3rd & 762 & 754 & 828 & 745 & 774 & 19 & 0.10 \\
\hline & 4 th & $1280^{\mathrm{ab}}$ & $953^{\mathrm{C}}$ & $1250^{\mathrm{b}}$ & $1325^{\mathrm{ab}}$ & $1391^{a}$ & 28 & 0.04 \\
\hline & 5 th & $1743^{\mathrm{ab}}$ & $1193^{c}$ & $1738^{\mathrm{ab}}$ & $1657^{\mathrm{b}}$ & $1922^{a}$ & 36 & $<0.001$ \\
\hline \multirow{3}{*}{ FE } & 3rd & 1.12 & 1.14 & 1.04 & 1.16 & 1.13 & 0.02 & 0.17 \\
\hline & $4^{\text {th }}$ & $1.27^{\mathrm{ab}}$ & $1.36^{\mathrm{a}}$ & $1.32^{\mathrm{a}}$ & $1.25^{\mathrm{ab}}$ & $1.12^{\mathrm{b}}$ & 0.04 & 0.01 \\
\hline & $5^{\text {th }}$ & $1.48^{\mathrm{b}}$ & $1.92^{\mathrm{a}}$ & $1.52^{\mathrm{b}}$ & $1.47^{\mathrm{b}}$ & $1.36^{\mathrm{b}}$ & 0.05 & $<0.001$ \\
\hline
\end{tabular}

The results of the relative weight of viscera in response to heat stress and supplementation of $\beta$-GOS are presented in Table 3. Heat exposure reduced $(P<0.05)$ the relative weights of liver, bursa of Fabricius and small intestine in HSCT compared with the TNZ group. Supplementation of $\beta$-GOS increased $(P<0.05)$ the relative weight of the bursa of Fabricius and liver in the HS + 0.5\% $\beta$-GOS group and the small intestine in $\mathrm{HS}+0.1 \% \beta$-GOS, HS $+0.2 \% \beta$-GOS, and $\mathrm{HS}+0.5 \% \beta$-GOS groups compared with the HSCT group (Table 3). 
Table 3 Effects of heat stress and $\beta$-galacto-oligosaccharides on relative weights of viscera in broilers

\begin{tabular}{|c|c|c|c|c|c|c|c|}
\hline \multirow[b]{2}{*}{ Parameter } & \multicolumn{5}{|c|}{ Groups* } & \multirow{2}{*}{$\begin{array}{l}\text { Pooled } \\
\text { SEM }\end{array}$} & \multirow{2}{*}{$\begin{array}{c}\mathrm{P}- \\
\text { value }\end{array}$} \\
\hline & TNZ & HSCT & HS+0.1\% $\beta-G O S$ & $\mathrm{HS}+0.2 \% \beta-G O S$ & $\mathrm{HS}+0.5 \% \beta-\mathrm{GOS}$ & & \\
\hline Liver & $2.4^{\mathrm{ab}}$ & $2.3^{\mathrm{b}}$ & $2.6^{\mathrm{ab}}$ & $2.4^{\mathrm{ab}}$ & $2.7^{\mathrm{a}}$ & 0.10 & 0.01 \\
\hline Spleen & 0.09 & 0.08 & 0.08 & 0.07 & 0.08 & 0.005 & 0.50 \\
\hline Gizzard & 3.2 & 2.8 & 2.9 & 2.5 & 3.3 & 0.10 & 0.14 \\
\hline Pancreas & 0.3 & 0.2 & 0.1 & 0.2 & 0.2 & 0.01 & 0.06 \\
\hline Heart & 0.4 & 0.5 & 0.5 & 0.4 & 0.5 & 0.01 & 0.25 \\
\hline Bursa & $0.07^{\mathrm{ab}}$ & $0.06^{b}$ & $0.07^{\mathrm{ab}}$ & $0.07^{\mathrm{ab}}$ & $0.08^{\mathrm{a}}$ & 0.05 & 0.04 \\
\hline Small intestine & $2.8^{\mathrm{b}}$ & $2.6^{c}$ & $3.3^{\mathrm{ab}}$ & $3.5^{\mathrm{ab}}$ & $4.1^{\mathrm{a}}$ & 0.20 & $<0.001$ \\
\hline Caecum & 0.69 & 0.72 & 0.77 & 0.74 & 0.78 & 0.15 & 0.06 \\
\hline
\end{tabular}

${ }^{\star \star}$ TNZ: control group; HSCT: heat stress control group; HS + 0.1\% $\beta$-GOS (0.1\% $\beta$-GOS-supplemented heat-stressed group); HS + $0.2 \% \beta$-GOS (0.2\% $\beta$-GOS-supplemented HS group) and HS + 0.5\% $\beta$-GOS (0.5\% $\beta$-GOS-supplemented heat-stressed group)

Values represent means of five replicates

${ }^{\mathrm{a}, \mathrm{b}, \mathrm{c}}$ Means within a row lacking a common superscript differ significantly at $(P<0.05)$

The results of the intestinal morphometric parameters in response to heat stress and supplementation of $\beta$-GOS are presented in Table 4.

Table 4 Effects of heat stress and $\beta$-galacto-oligosaccharides on histomorphometry of the small intestine in broilers

\begin{tabular}{|c|c|c|c|c|c|c|c|}
\hline \multirow{2}{*}{ Parameters } & \multicolumn{5}{|c|}{ Groups* } & \multirow{2}{*}{$\begin{array}{l}\text { Pooled } \\
\text { SEM }\end{array}$} & \multirow{2}{*}{$\begin{array}{c}\text { P- } \\
\text { value }\end{array}$} \\
\hline & TNZ & HSCT & HS+0.1\% $\%$-GOS & $\mathrm{HS}+0.2 \% \beta-G O S$ & HS $+0.5 \% \beta-G O S$ & & \\
\hline \multicolumn{8}{|l|}{ Duodenum } \\
\hline $\mathrm{VH}(\mu \mathrm{m})$ & $944^{\text {cd }}$ & $837^{d}$ & $1239^{a}$ & $1130^{\mathrm{ab}}$ & $1009^{b c}$ & 31 & $<0.001$ \\
\hline $\mathrm{VW}(\mu \mathrm{m})$ & $118^{\mathrm{b}}$ & $91^{\mathrm{b}}$ & $175^{\mathrm{a}}$ & $95^{\mathrm{b}}$ & $97^{b}$ & 6 & $<0.001$ \\
\hline $\operatorname{VSA}\left(\mu \mathrm{m}^{2}\right)$ & $349706^{b}$ & $240147^{b}$ & $679099^{a}$ & $344550^{b}$ & $307120^{b}$ & 28448 & $<0.001$ \\
\hline $\mathrm{CD}(\mu \mathrm{m})$ & $192^{\mathrm{a}}$ & $102^{\mathrm{C}}$ & $185^{\mathrm{ab}}$ & $145^{\mathrm{b}}$ & $147^{\mathrm{b}}$ & 8 & $<0.001$ \\
\hline $\mathrm{VH}: \mathrm{CD}$ & $9.6^{\mathrm{a}}$ & $4.9^{b}$ & $7.3^{\mathrm{a}}$ & $8.0^{\mathrm{a}}$ & $7.3^{\mathrm{a}}$ & 0.40 & 0.03 \\
\hline \multicolumn{8}{|l|}{ Jejunum } \\
\hline $\mathrm{VH}(\mu \mathrm{m})$ & $1012^{b}$ & $854^{\mathrm{c}}$ & $843^{c}$ & $1150^{\mathrm{a}}$ & $1254^{\mathrm{a}}$ & 30 & $<0.001$ \\
\hline $\mathrm{VW}(\mu \mathrm{m})$ & $1012^{b}$ & $854^{\circ}$ & $843^{c}$ & $1150^{\mathrm{a}}$ & $1254^{\mathrm{a}}$ & 30 & $<0.001$ \\
\hline $\operatorname{VSA}\left(\mu \mathrm{m}^{2}\right)$ & $500258^{b}$ & $248727^{c}$ & $274342^{c}$ & $563871^{\mathrm{b}}$ & $754417^{\mathrm{a}}$ & 32205 & $<0.001$ \\
\hline $\mathrm{CD}(\mu \mathrm{m})$ & $211^{\mathrm{b}}$ & $101^{\mathrm{c}}$ & $195^{b}$ & $299^{a}$ & $289^{a}$ & 13 & $<0.001$ \\
\hline $\mathrm{VH}: \mathrm{CD}$ & $10^{\mathrm{a}}$ & $4.1^{\mathrm{b}}$ & $5^{\mathrm{b}}$ & $4^{b}$ & $5^{\mathrm{b}}$ & 0.3 & $<0.001$ \\
\hline \multicolumn{8}{|l|}{ Ileum } \\
\hline $\mathrm{VH}(\mu \mathrm{m})$ & $950^{b}$ & $815^{\mathrm{c}}$ & $953^{b}$ & $975^{b}$ & $1180^{\mathrm{a}}$ & 20 & $<0.001$ \\
\hline $\mathrm{VW}(\mu \mathrm{m})$ & $132^{b}$ & $74^{\mathrm{c}}$ & $182^{\mathrm{a}}$ & $164^{\mathrm{a}}$ & $176^{\mathrm{a}}$ & 6 & $<0.001$ \\
\hline $\operatorname{VSA}\left(\mu \mathrm{m}^{2}\right)$ & $392837^{c}$ & $186814^{d}$ & $541711^{\mathrm{b}}$ & $503870^{b}$ & $651797^{a}$ & 23913 & $<0.001$ \\
\hline $\mathrm{CD}(\mu \mathrm{m})$ & $227^{\mathrm{a}}$ & $149^{c}$ & $181^{\mathrm{b}}$ & $201^{\mathrm{b}}$ & $235^{\mathrm{a}}$ & 6 & $<0.001$ \\
\hline $\mathrm{VH}: \mathrm{CD}$ & $6^{a}$ & $4^{c}$ & $4.8^{\mathrm{b}}$ & $4.8^{\mathrm{b}}$ & $5^{b}$ & 0.17 & 0.001 \\
\hline
\end{tabular}

${ }^{*}$ TNZ: control group; HSCT: heat stress control group; HS + 0.1\% $\beta$-GOS $(0.1 \% \beta$-GOS-supplemented heat-stressed group $) ; \mathrm{HS}+0.2 \%$ $\beta$-GOS (0.2\% $\beta$-GOS-supplemented HS group) and HS + 0.5\% $\beta$-GOS ( $0.5 \% \beta$-GOS-supplemented heat-stressed group)

VH: villus height; VW: villus width; VSA: villus surface area; $C D$ : crypt depth; VH : CD: villus height : crypt depth ratio

Values represent means of five replicates

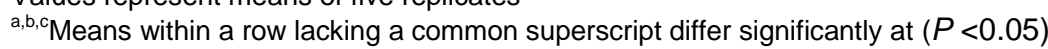


Heat exposure reduced $(P<0.05)$ villus height and crypt depth in all segments of the small intestine and villus width in the jejunum compared with the TNZ group. Furthermore, it reduced $(P<0.05)$ intestinal villus surface area in the duodenum, jejunum and ileum compared with the TNZ group.

Compared with the HSCT group, all the dietary inclusion of $\beta$-GOS increased $(P<0.05)$ the villus height and villus surface area of the duodenum, whereas the villus width and crypt depth of the duodenum increased $(P<0.05)$ with only $0.1 \% \beta$-GOS supplementation. In the jejunum, villus height, villus width, and villus surface area increased $(P<0.05)$ with $0.2 \%$ and $0.5 \% \quad \beta$-GOS supplementation, and crypt depth increased $(P<0.05)$ with all the dietary inclusions of $\beta$-GOS compared with the HSCT group. In the ileum, villus height, crypt depth and villus surface area increased $(P<0.05)$ with all dietary inclusion of $\beta$-GOS supplementation compared with the HSCT group.

The results of the IEL count of the small intestine in response to heat stress and supplementation of $\beta$ GOS are presented in Table 5. Heat exposure reduced $(P<0.05)$ the IEL count of the small intestine compared with the TNZ group. Dietary supplementation of $0.1 \% \beta$-GOS increased $(P<0.05)$ the IEL count, and $0.2 \%$ and $0.5 \% \beta$-GOS reduced $(P<0.05)$ the IEL count of the small intestine compared with the HSCT group.

Table 5 Effects of heat stress and $\beta$-galacto-oligosaccharides on intraepithelial lymphocyte count of small intestine in broilers

\begin{tabular}{lccccccc}
\hline \multirow{2}{*}{ Parameters } & \multicolumn{5}{c}{ Groups $^{*}$} & \multirow{2}{*}{ Pooled SEM } & P-value \\
\cline { 2 - 5 } & TNZ & HSCT & HS+0.1\% $\beta$-GOS & HS+0.2\% $\%$-GOS & HS+0.5\% $\%$-GOS & & \\
\hline \multirow{2}{*}{ Duodenum } & $108^{\mathrm{a}}$ & $50^{\mathrm{c}}$ & $69^{\mathrm{b}}$ & $49^{\mathrm{c}}$ & $49^{\mathrm{c}}$ & 3.8 & $<0.001$ \\
Jejunum & $79^{\mathrm{a}}$ & $47^{\mathrm{c}}$ & $68^{\mathrm{b}}$ & $34^{\mathrm{d}}$ & $35^{\mathrm{d}}$ & 2.9 & $<0.001$ \\
lleum & $85^{\mathrm{a}}$ & $35^{\mathrm{d}}$ & $60^{\mathrm{b}}$ & $45^{\mathrm{c}}$ & $38^{\mathrm{cd}}$ & 2.9 & $<0.001$
\end{tabular}

${ }^{*}$ TNZ: control group; HSCT: heat stress control group; HS + 0.1\% $\beta$-GOS ( $0.1 \% \beta$-GOS-supplemented heat-stressed group); HS + 0.2\% $\beta$-GOS $(0.2 \% \beta$-GOS-supplemented HS group) and HS + 0.5\% $\beta$-GOS ( $0.5 \% \beta$-GOS-supplemented heat-stressed group)

Values represent means of five replicates

${ }_{\mathrm{a}, \mathrm{b}, \mathrm{c}, \mathrm{d}}$ Means within a row lacking a common superscript differ significantly at $(P<0.05)$

The results of the acidic mucin goblet cell count of the small intestine in response to heat stress and supplementation of $\beta$-GOS are presented in Table 6 . In the small intestine, histochemistry of the goblet cell revealed cells having acidic glycoprotein content. Neither heat stress nor supplementation influenced the acidic mucin goblet cell count in the duodenum. In the jejunum, exposure to heat stress increased $(P<0.05)$ the count of acidic mucin goblet cells, while in the ileum their count was decreased $(P<0.05)$ in the HSCT compared with the TNZ group. Dietary supplementation of $0.2 \%$ and $0.5 \% \beta$-GOS reduced the count of acidic mucin containing goblet cells in the jejunum and ileum compared with the HSCT group.

Table 6 Effects of heat stress and $\beta$-galacto-oligosaccharides supplementation on acidic mucin goblet cells of small intestine in broilers

\begin{tabular}{|c|c|c|c|c|c|c|c|}
\hline \multirow{2}{*}{ Parameters } & \multicolumn{5}{|c|}{ Groups* } & \multirow{2}{*}{ Pooled SEM } & \multirow{2}{*}{ P-value } \\
\hline & TNZ & HSCT & HS+0.1\% $\%$-GOS & $\mathrm{HS}+0.2 \% \beta-\mathrm{GOS}$ & $\mathrm{HS}+0.5 \% \beta-\mathrm{GOS}$ & & \\
\hline Duodenum & 75 & 82 & 72 & 109 & 64 & 5.3 & 0.06 \\
\hline Jejunum & $79^{b}$ & $107^{a}$ & $100^{a}$ & $77^{\mathrm{b}}$ & $75^{\mathrm{b}}$ & 3.2 & 0.001 \\
\hline Ileum & $94^{\mathrm{a}}$ & $58^{b}$ & $63^{b}$ & $28^{d}$ & $38^{c}$ & 3.5 & $<0.001$ \\
\hline
\end{tabular}




\section{Discussion}

The present study demonstrates the effects of various dietary inclusions of $\beta$-GOS originating from Lactobacillus reuteri L103 on the growth performance, organ development, intestinal microarchitecture, intraepithelial lymphocytes, and goblet cell differentiation during heat stress in broilers. In broiler production, exposure of bird to heat stress results in economic losses and welfare issues. To achieve optimal production targets, particularly during the summer months, the temperature of the shed must be maintained in a narrow range of $18{ }^{\circ} \mathrm{C}$ to $22{ }^{\circ} \mathrm{C}$, which is costly (Yoo et al., 2016). Antibiotics were utilized in the poultry industry to reduce the adverse effects of environmental stress, but their use in animal feed was banned by the EU in 2006 (Skoufos et al., 2016). Therefore, many studies have explored dietary supplements that could not only improve the gut health of birds, but also replace growth promoting antibiotics. Prebiotics are indigestible oligosaccharides that affect the host beneficially (Alloui et al., 2013) and provide an alternative approach to antibiotic growth promoters.

The results of the present study revealed that exposure to heat stress negatively influenced all the growth parameters of broilers, including feed consumption, bodyweight, and feed efficiency. The results of the current study are in alignment with the information that has been consistently observed for these parameters during heat exposure, as reviewed by Lara \& Rostagno (2013). Reduction of feed consumption is a heat stress-induced adaptation of birds to generate less heat, mediated via decreased triiodothyronine and increased corticosterone. Corticosterone acts on hypothalamic nuclei and reduces appetite. This behavioural and hormonal change is primarily responsible for reduction in weight gain and poor performance during heat stress (Quinteiro-Filho et al., 2010). Other contributing factors may be inefficient digestion and absorption owing to intestinal injuries, and reduction in weight of liver and pancreas during heat exposure (Hasan et al., 2014). During final two weeks, all dietary inclusions of $\beta$-GOS improved the feed consumption, bodyweight, and feed efficiency of heat-stressed broilers. In general, prebiotic-induced improvement in growth performance, including bodyweight and feed efficiency of the heat-stressed broilers, could be compared with the studies of Silva et al. (2010), Haldar et al. (2011), and Hasan et al. (2014). Specifically for $\beta$-GOS, the authors could find few reports indicating the variable influence of $\beta$-GOS on growth performance in broilers in thermoneutral situations. Biggs et al. (2007) did not observe any improvement in the performance of $\beta$-GOS-supplemented birds. Earlier reports demonstrated that the effects of the prebiotics on the performance of broilers are inconsistent (Biggs et al., 2007). This might be because of the composition of prebiotics, dose rates and the experimental situations, that is, thermoneutral versus heat stress, as it was believed that prebiotics work optimally under stressed conditions (Baurhoo et al., 2007). The $\beta$-GOS-induced improvement observed in the present study may be ascribed to the ability of prebiotics to promote healthy intestinal flora, immunomodulation (Vendrig et al., 2013) improvement in the structural integrity of the intestinal barrier (Varasteh et al., 2015), and correction of adrenal dysfunction.

Exposure to heat stress reduced the relative weights of liver, small intestine and bursa of Fabricius in broilers. Regarding the effects of heat stress on immune organs, the findings of the current study reiterated those of previous reports (Muniz et al., 2006; Quinteiro-Filho et al., 2010). Decreased feed consumption, disturbance of metabolism owing to decreased triiodothyronine levels and mal-absorption because of intestinal injuries in general might be responsible for reducing the relative weights of viscera during heat stress. In addition, increase in the corticosterone-induced apoptosis of lymphocytes and reduction in the parenchyma of lymphoid organs during heat stress might have contributed to the decreased relative weight of bursa of Fabricius in the current study (Muniz et al., 2006). All the dietary inclusions of $\beta$-GOS improved the relative weights of intestines, bursa of Fabricius and liver in heat-stressed broilers. Improvement in relative weights of lymphoid organs and intestine in response to other prebiotics during heat stress was published by Haung et al. (2007), but currently the authors lack sufficient literature that explains the effects of $\beta$-GOS on the relative weight of viscera during thermoneutral and heat-stress situations in broilers. However, Vendrig et al. (2013) indicated its immunomodulatory properties, which might be responsible for the increase in relative weight of bursa of Fabricius. Varasteh et al. (2015) reported an improvement in jejunal barrier function of GOS-fed heat-stressed broilers, which might have contributed to the improved microarchitecture and increased relative weight of the small intestine. Improvements in feed efficiency and hormonal status were also observed with $\beta$-GOS supplementation. Therefore, a positive relationship may be assumed to exist between growth performance and increased relative weight of the small intestine and immune organs.

The intestinal morphometric parameters, especially the villus surface area and VH : CD are regarded as indicators of gut health (Haldar et al., 2011) and are adversely affected by heat stress (Silva et al., 2010). During heat exposure, the results of the current study revealed a significant decrease in villus surface area of the small intestine, which was a reflection of a decrease in villus height. Moreover, the VH : CD was lower during heat stress, which again shows that the intestinal environment was unfavourable for the absorption of nutrients owing to the shortening of villus height (Yoo et al., 2016). These findings are in agreement with the results of the authors' previous study (Ashraf et al., 2013) and those of other heat-stress trials conducted in 
broilers (Silva et al., 2010; Haldar et al., 2011; Song et al., 2014). Intestinal mucosal lesions are among the initial manifestations of stress, and are attributed to ischemia of the gut wall, production of reactive oxygen species (Lambert et al., 2009), expression of heat shock proteins, and increases in pro-inflammatory cytokines and intestinal microbial dysbiosis (Song et al., 2014). Shedding of epithelial cells in response to these changes might have accounted for the decreased villus surface area and lower VH : CD observed in the current study (Rivera et al., 2011). An increase in villus surface area was observed in the duodenum with $0.1 \% \beta$-GOS, in the jejunum with 0.2 and $0.5 \% \beta$-GOS, and in the ileum in all the $\beta$-GOS-supplemented heatstressed groups. The $\mathrm{VH}$ : $\mathrm{CD}$ ratio was also improved in the duodenum and ileum of these groups. These findings suggest that the supplementation of $\beta$-GOS neutralized the effects of heat stress on small intestinal villus crypt units. The protective effects of $\beta$-GOS on the jejunal mucosal barrier during heat stress (Varasteh et al., 2015), Salmonella typhimurium infection (Searle et al., 2010) and toxin exposure (Akbari et al., 2015) had been observed earlier. Dietary supplementation of $\beta$-GOS has proved to be beneficial for increasing the number of Lactobacillus and Bifidobacterium and for reducing the attachment of enteropathogens to the intestinal mucosa. (Varasteh et al., 2015). Moreover, the antioxidant properties of $\beta$-GOS and microbiota independent interactions of the $\beta$-GOS with enterocytes have been confirmed recently (Varasteh et al., 2015). These factors individually and in combination might have resulted in an improvement in villus surface area in the current situations.

Intra epithelia lymphocytes are an important effector component of the gut-associated lymphoid tissue against the luminal antigens in the intestine (McDonald, 1999). Exposure to heat stress resulted in a decline in the IEL count in all segments of the small intestine. Similar observations were made in broilers by Deng et al., (2012). However, these results are in contrast with those reported by Ashraf et al. (2013) and QuinteiroFilho et al. (2010). In general, stress response is an interplay between many neural and hormonal factors (Borsoi et al., 2015) and duration, type and frequency of the stress are important stress modifiers. Therefore, variations among the results of various studies may seem highly plausible (Quinteiro-Filho et al., 2015). The difference in the results of the current two heat stress trials may be explained in terms of the types of IELs triggered by different pathogens. IELs have two types of T-cell receptors (TCR), namely $\alpha \beta$ and $\gamma \delta^{+}$. IELs with $y \delta^{+} T C R$ induce or down-regulate inflammation induced by IELs with $\alpha \beta T C R$. Although the authors did not assess the types of infections or microbes during the trials, they may speculate that there was a difference in types of microbes in previous situations (intracellular pathogens) and in current ones (mucosaattaching pathogens) which resulted in variations. In the current experiment, decreased IEL count may be interlinked with hyper-activation of the HPA axis and increased cortisol (data not published). Moreover, stress hormones not only inhibit the production of pro-inflammatory cytokines, but also stimulate the production of anti-inflammatory cytokines (Sternberg \& Licinio, 1995), along with apoptosis of IELs (JarilloLuna et al., 2008), which might have contributed to the decreased IEL count. The addition of $0.1 \% \beta$-GOS increased the IELs count in all intestinal segments of the heat-stressed broilers. However, the authors could not observe a dose-dependent effect of this supplementation; nor could it restore the pre-heat stress count of IELs. A review of the literature reveals the variable effects of prebiotics on the immune system, ranging from immune stimulation (Gaskins et al., 1996; Schley \& Field, 2002) to immune suppression (Janardhana et al., 2009). These effects may be related to the ability of prebiotics - including GOS - to produce pro- and antiinflammatory cytokines (Searle et al., 2010). The exact mechanism governing the production of cytokines (pro- and anti-inflammatory) and the predominant effects of only one type of cytokine during supplementation need further explanation. Multiple reasons have been put forward to explain the variable effects of prebiotics on the immune system, for example the dose, nature and origin of the prebiotics, and the presence or absence of stress. (Baurhoo et al., 2007; Callaway et al., 2012). However, the phenomenon of prebioticinduced immunomodulation still needs elucidation. The rise in the IEL count of supplemented heat-stressed birds during the current trial may be attributed to an increase in the population of probiotic organisms and fractions of bacteria in the GOS product that are responsible for stimulating and recruiting the immune cells at intestinal surface (Deng et al., 2012) and may be regarded as physiological inflammation at the surface of gut.

Goblet cells are mucin-producing cells that provide resistance to the colonization of pathogenic bacteria and hence are important components of the intestinal barrier. Goblet cells with acidic glycoproteins are mature cells that confer better resistance against pathogens (Deplancke \& Gaskins, 2000). Heat stress exposure did not influence the count of acidic mucin goblet cells in the duodenum, whereas the jejunal and ileal acidic mucin goblet cell counts were increased and decreased, respectively. Comparable effects of stress on the count of goblet cells have been reported previously (Söderholm \& Perdue, 2001; Deng et al., 2012; Ashraf et al., 2013). An increase in the number of these acidic mucin goblet cells in the jejunum might be translated as better preparedness of mucosa in response to the indigenous microbial shift during heat stress. However, this effect could not be observed in the ileum. Since the susceptibility of intestinal segments varies towards heat stress, differences in their responses in terms of acidic mucin goblet cells count seem 
probable (Varasteh et al., 2015). The acidic mucin goblet cells of the ileum might have released their contents in response to increased pathogen load during heat stress, leaving the goblet cell empty at the time of staining (Ashraf et al., 2013). Supplementation of $\beta$-GOS $(0.2 \%$ and $0.5 \%)$ further reduced the count of acidic mucin goblet cells in the jejunum and ileum. In contrast with these results, various studies have reported an increase in the count of goblet cells with the supplementation of various pre- and probiotics (Baurhoo et al., 2007; Ashraf et al., 2013). To the best of the authors' knowledge, the effects of $\beta$-GOS on the quantitative and qualitative aspects of acidic mucin goblet cell count in broilers have not been reported. Therefore, at present, it may be difficult to explain the exact mechanism behind this decrease. However, it may be hypothesized that $\beta$-GOS might have potentiated the effects of heat stress on the release of mucins for counteracting pathogens, as reported by Searle et al. (2010).

\section{Conclusion}

The study showed that exposure to heat stress impaired growth performance, organ development, and intestinal mucosal barrier components in broilers. Dietary supplementation of $\beta$-GOS $(0.2 \%$ and $0.5 \%)$ ameliorated these detrimental effects of heat stress, along with partial immune stimulation.

\section{Acknowledgements}

This work was sponsored by Higher Education Commission, Pakistan.

\section{Authors' Contributions}

All the authors contributed and commented on early and final versions of manuscript. SA,HZ, HR, and SY conceived and designed the experiment. SA and AS carried out the experiment. SM, MMU, and HFR assisted in laboratory analysis. SA, HZ, SY, and HFR helped in manuscript preparation.

\section{Conflict of Interest Declaration}

The authors have no conflict of interest to declare.

\section{References}

Akbari, P., Braber, S., Alizadeh, A., Verheijden, K.A., Schoterman, M.H., Kraneveld, A.D., Garssen, J. \& Fink-Gremmels, J., 2015. Galacto-oligosaccharides protect the intestinal barrier by maintaining the tight junction network and modulating the inflammatory responses after a challenge with the mycotoxin deoxynivalenol in human caco-2 cell monolayers and b6c3f1 mice. J. Nutr. 145, 1604-1613.

Alloui, M.N., Szczurek, W. \& Swiatkiewicz, S., 2013. The usefulness of prebiotics and probiotics in modern poultry nutrition: A review. Ann. Anim. Sci. 13, 17-32.

Ashraf, S., Zaneb, H., Yousaf, M., Ijaz, A., Sohail, M., Muti, S., Usman, M., ljaz, S. \& Rehman, H., 2013. Effect of dietary supplementation of prebiotics and probiotics on intestinal microarchitecture in broilers reared under cyclic heat stress. J. Anim. Physiol. Anim. Nutr. 97, 68-73.

Bancroft, J.D., Floyd, A.D. \& Suvarna, S.K., 2013. Bancroft's theory and practice of histological techniques. 7th ed. Churchill Livingstone. New York, USA. 173-180 pp.

Baurhoo, B., Phillip, L. \& Ruiz-Feria, C.A., 2007. Effect of purified lignin and mannan-oligosaccharide on Salmonella colonization of the chicken intestine. Poult. Sci. 70, 2433-2438.

Biggs, P., Parsons, C. \& Fahey, G., 2007. The effects of several oligosaccharides on growth performance, nutrient digestibilities, and cecal microbial populations in young chicks. Poult. Sci. 86, 2327-2336

Borsoi, A., Calefi, A.S., Ferreira, A.J.P. \& Astolfi-Ferreira, C.S., 2015. Effects of cold stress and Salmonella Heidelberg infection on bacterial load and immunity of chickens. Avian Pathol. 44, 490-497.

Bruno-Barcena, J.M. \& Azcarate-Peril, M.A., 2015. Galacto-oligosaccharides and colorectal cancer: Feeding our intestinal probiome. J. Funct. Foods. 12, 92-108.

Burkholder, K.M., Thompson, K.L., Einstein, M.E., Applegate, T.J. \& Patterson, J.A., 2008. Influence of stressors on normal intestinal microbiota, intestinal morphology, and susceptibility to Salmonella Enteritidis colonization in broilers. Poult. Sci. 87, 1734-1741.

Callaway, T.R., Edrington, T.S., Harvey, R.B., Anderson, R.C. \& Nisbet, D.J., 2012. Prebiotics in food animals, a potential to reduce foodborne pathogens and disease. Rom. Biotechnol. Lett. 17, 7808-7816.

Decuypere, E., Buyse, J. \& Buys, N., 2000. Ascites in broiler chickens: Exogenous and endogenous structural and functional causal factors. World Poult. Sci. J. 56, 367-377.

Deng, W., Dong, X.F., Tong, J.M. \& Zhang, Q., 2012. The probiotic Bacillus licheniformis ameliorates heat stressinduced impairment of egg production, gut morphology, and intestinal mucosal immunity in laying hens. Poult. Sci. 91, 575-582.

Deplancke, B. \& Gaskins, H.R., 2000. Microbial modulation of innate defense: Goblet cells and the intestinal mucus layer1-3. Am. J. Clin. Nutr. 73, 1131-1141.

Gaskins, H.R., Mackie, R.I., May, T. \& Garleb, K.A., 1996. Dietaryfructo-oligosaccharide modulates large intestinal inflammatoryresponses to clostridium difficile in antibiotic-compromised mice. Microb. Ecol. Health. 9, 157-166.

Haldar, S., Ghosh, T.K., \& Bedford, M.R., 2011. Effects of yeast (Saccharomyces cervisiae) and yeast protein concentrate on production performance of broiler chickens exposed to heat stress and challenged with Salmonella enteritidis. Anim. Feed. Sci. Tech. 169, 61-71. 
Hasan, S., Hossain, M.M., Miah, A., \& Bhuiyan, M.E.R., 2014. Influences of prebiotic on growth performance and hematobiochemical parameters in broiler during heat stress. Bangl. J. Vet. Med. 12, 121-125.

Huang, R.L., Deng, Z.Y., Yang, C., Yin, Y.L., Xie, M.Y., Wu, G.Y., Li, T.J., Li L.L., Tang Z.R., Kang, P., Hou, Z.P., Deng, D., Xiang, H., Kong, X.F. \& Guo, Y.M., 2007. Dietary oligochitosan supplementation enhances immune status of broilers. J. Sci. Food Agri. 87, 153-159.

Huff, G.R., Huff, W.E., Rath, N.C., El-Gohary, F.A., Zhou, Z.Y. \& Shini, S., 2015. Efficacy of a novel prebiotic and a commercial probiotic in reducing mortality and production losses due to cold stress and Escherichia coli challenge of broiler chicks. Poult. Sci. 94, 918-926.

Janardhana, V., Broadway, M.M., Bruce, M.P., Lowenthal, J.W., Geier, M.S., Hughes, R.H. \& Bean, A.G.D., 2009. Prebiotics modulate immune responses in gut-associated lymphoid tissue of chickens. J. Nutr. 139, 1404-1409.

Jarillo-Luna, A., Rivera-Aguilar, V., Martìnez-Carrillo, B.E., Barbosa-Cabrera, E., Garfias, H.R. \& Campos-Rodríguez, R. 2008. Effect of restraint stress on the population of intestinal intraepithelial lymphocytes in mice. Brain Behav. Immun. 22, 265-275.

Kazmi D.H., Li, J., Rasul, G., Tong, J., Ali, G., Cheema, S. B., Liu, L., Gemmer, M. \& Fischer, T., 2015. Statistical downscaling and future scenario generation of temperatures for Pakistan Region. Theor. Appl. Climatol. 120, 341350.

Kolida, S. \& Gibson, G.R., 2011.Synbiotics in health and disease. Annu. Rev. Food Sci. Techno. 2, 373-393.

Lambert, G.P., 2009. Stress-induced gastrointestinal barrier dysfunction and its inflammatory effects. J. Anim. Sci. 87, 101-108

Lara, J. \& Rostagno, M.H., 2013. Impact of heat stress on poultry production. Animals. 3, 356-369.

McDonald, V., 1999. Gut intraepithelial lymphocytes and immunity to coccidia. Parasitol. Today. 15, 483-487.

Muniz, E.C., Fascina, V.B., Pires, P.P., Carrijo, A.S. \& Guimarães, E.B., 2006. Histomorphology of bursa of Fabricius: Effects of stock densities on commercial broilers. Rev. Bras. Ciência. Avícola. 8, 217-220.

NRC, 1994. Nutrient requirements of poultry. Ninth revised edition. National Academy Press, Washington D.C.

Olsen, R.E., Sundellb, T.K., Mayhewc, T.M., Myklebustd, R. \& Ringøa, E., 2005. Acute stress alters intestinal function of rainbow trout, Oncorhynchus mykiss (Walbaum). Aquaculture. 250, 480-495.

Quinteiro-Filho, W.M., Ribeiro, A., Paula, F.V., Pinheiro, L., Sakai, M., Sa, L.R.M., Ferreira, A.J.P. \& Palermo-Neto, J., 2010. Heat stress impairs performance parameters, induces intestinal injury, and decreases macrophage activity in broiler chickens. Poult. Sci. 89, 1905-1914.

Quinteiro-Filho, W.M., Rodrigues, M.V., Ribeiro, A., Ferraz-de-Paula, V., Pinheiro, M.L., Sá, L.R.M., Ferreira, A.J.P. \& Palermo-Neto,J., 2015. Acute heat stress impairs performance parameters and induces mild intestinal enteritis in broiler chickens: Role of acute hypothalamic-pituitary-adrenal axis activation. J. Anim. Sci. 90, 1986-1994.

Rivera, L.R., Thacker, M., Pontell, L., Cho, H.J. \& Furness, J.B., 2011. Deleterious effects of intestinal ischemia/reperfusion injury in the mouse enteric nervous system are associated with protein nitrosylation. Cell Tissue Res. 344, 111-123.

Schley, P.D. \& Field, C.J., 2002. The immune-enhancing effects of dietary fibres and prebiotics. Brit. J. Nutr. 87, $221-$ 230.

Searle, L.E.J., Cooley, W.A., Jones, G., Nunez, A., Crudgington, B., Weyer, U., Dugdale, A.H., Tzortzis, G., Collins, J.W., Woodward, M.J. \& Ragione, R.M.L., 2010. Purified galactooligosaccharide, derived from a mixture produced by the enzymic activity of Bifidobacterium bifidum, reduces Salmonella enterica serovar Typhimurium adhesion and invasion in vitro and in vivo. J. Med. Microbiol. 59, 1428-1439.

Silva, V.K., Silva, T.D.J.D., Gravena, R.A., Marques, R.H., Hada, F.H. \& Barbosa-de-Moraes, V.M., 2010. Yeast extract and prebiotic in pre-initial phase diet for broiler chickens raised under different temperatures. Rev. Bras. Zootec. 39, $165-174$.

Skoufos, I., Giannenas, I., Tontis, D., Bartzanas, T., Kittas, C., Panagakis, P. \& Tzora, A., 2016. Effects of oregano essential oil and attapulgite on growth performance, intestinal microbiota and morphometry in broilers. S. Afr. J. Anim. Sci. 46, 77-88.

Soderholm, J.D. \& Perdue, M.H., 2001. Stress and intestinal barrier function. Am. J. Physiol. Gastrointest. Liver Physiol. $280,7-13$

Solis-de-los Santos, F., Donoghue, A.M., Farnell, M.B., Huff, G.R., Huff, W.E. \& Donoghue, D.J., 2007. Gastrointestinal maturation is accelerated in turkey poults supplemented with a Mannan-oligosaccharide yeast extract (alphamune). Poult. Sci. 86, 921-930.

Song, J., Xiao, K., Ke, X.L., Jiao, L.F., Hu, C.H., Diao, Q.Y., Shi, B. \& Zou, X.T., 2014. Effect of a probiotic mixture on intestinal microflora, morphology, and barrier integrity of broilers subjected to heat stress. Poult. Sci. 93, 581-588.

Sternberg, E.M. \& licinio, J., 1995. Overview of neuroimmune stress interactions. Ann N.Y. Acad. Sci. 771, $363-371$.

Thrall, M.A., Dale, C. \& Baker Duane, L. E., 2004. Veterinary Hematology and Clinical Chemistry. Lippincott Williams \& Wilkins, Philadelphia, USA.

Varasteh, S., Braber, S., Akbari, P., Garssen, J. \& Gremmels, J.F., 2015. Differences in susceptibility to heat stress along the chicken intestine and the protective effects of galacto-oligosaccharides. PLoS ONE. 10, 1-18.

Vendrig, J.C., Coffeng, L.E. \& Fink-Gremmels, J., 2013. In vitro evaluation of defined oligosaccharide fractions in an equine model of inflammation. BMC Vet. Res. 9, 147-157.

Yoo, J., Yi, Y.J., Koo, B., Jung, S., Yoon, J.U., Kang, H.B., Lee, D.H. \& Heo, J.M., 2016. Growth performance, intestinal morphology, and meat quality in relation to alpha-lipoic acid associated with vitamin $C$ and $E$ in broiler chickens under tropical conditions. R. Bras. Zootec. 45,113-120. 